\title{
Small Poly-L-Lysines Improve Cationic Lipid-Mediated Gene Transfer in Vascular Cells in vitro and in vivo
}

\author{
Adam Golda $^{a}$ Jaroslav Pelisek ${ }^{a, c}$ Rainer Klocke ${ }^{a}$ Markus G. Engelmann ${ }^{b}$ \\ Pierre-Henri Rolland $^{d}$ Choukri Mekkaouid $^{d}$ Sigrid Nikola \\ ${ }^{a}$ Department of Cardiology and Angiology, University Hospital, Westfaelische Wilhelms University, Muenster, \\ ${ }^{b}$ Medical Department I, University Hospital, Grosshadern Ludwig Maximilian University, and \\ 'Department of Vascular Surgery, Klinikum rechts der Isar, Technical University Munich, Munich, Germany; \\ dLaboratory of Hemodynamics and Cardiovascular Mechanics, School of Medicine, Mediterranean University, \\ Marseille, France
}

\section{Key Words}

Gene transfer $\cdot$ Lipopolyplexes $\cdot$ Poly-L-lysine $\cdot$ Cationic

lipid · Smooth muscle cells

\begin{abstract}
The potential of two small poly-L-lysines (sPLLs), low molecular weight SPLL (LMW-L) containing 7-30 lysine residues and L18 with 18 lysine repeats, to enhance the efficiency of liposome-mediated gene transfer (GT) with cationic lipid DOCSPER [1,3-dioleoyloxy-2-( $\mathrm{N}^{5}$-carbamoyl-spermine)-propane] in vascular smooth muscle cells (SMCs) was investigated. Dynamic light scattering was used for determination of particle size. Confocal microscopy was applied for colocalization studies of sPLLs and plasmid DNA inside cells. GT was performed in proliferating and quiescent primary porcine SMCs in vitro and in vivo in porcine femoral arteries. At low ionic strength, sPLLs formed small complexes with DNA (50$100 \mathrm{~nm})$. At high ionic strength, large complexes ( $>1 \mu \mathrm{m})$ were observed without any significant differences in particle size between lipoplexes (DOCSPER/DNA) and lipopolyplexes (DOCSPER/sPLL/DNA). Both sPLLs were colocalized with DNA inside cells $24 \mathrm{~h}$ after transfection, protecting DNA against degradation. DOCSPER/sPLL/DNA formulations enhanced GT in vitro up to 5 -fold, in a porcine model using lo-
\end{abstract}

cal periadventitial application up to 1.5 -fold. Both sPLLs significantly increased liposome-mediated GT. Poly-L-lysine L18 was superior to LMW-L since it enabled maximal GT at a 10fold lower concentration. Thus, sPLLs may serve as enhancers for GT applications in SMCs in vitro and in vivo using local delivery.

Copyright $\odot 2007$ S. Karger AG, Basel

\section{Introduction}

Conventional drug therapy has limitations that can be overcome using gene therapy [1]. The advantages of gene therapy are the long-lasting effects, tolerance of gene constructs, the possibility to direct the expression of the therapeutic gene to certain cell types and low unwanted side effects, in particular using local delivery [2]. Gene therapy represents, among other applications, a promising alternative for treatment of vascular disease, e.g., the prevention of restenosis following angioplasty. Neointima formation after balloon dilatation is caused mainly by proliferation of smooth muscle cells (SMCs) and matrix formation [3]. Thus, inhibition of SMC proliferation also has a suppressive effect on restenosis $[4,5]$. The arterial wall is accessible by drug delivery catheters that facilitate

\section{KARGER}

Fax +4161306 1234 E-Mail karger@karger.ch www.karger.com
(C) 2007 S. Karger AG, Basel $1018-1172 / 07 / 0444-0273 \$ 23.50 / 0$

Accessible online at: www.karger.com/jvr
Prof. Dr. Sigrid Nikol

Department of Cardiology and Angiology

Medizinische Klinik und Poliklinik C, University of Münster

Albert-Schweitzer-Strasse 33, DE-48129 Münster (Germany)

Tel. +49 251834 8501, Fax +49251834 5101, E-Mail nikol@uni-muenster.de 
the local deposition of active agents [6]. It has been shown that perivascular events significantly affect neointima: adventitial irritation results in focal intimal thickening and markers applied to the adventitia can be visualized in the intima $[7,8]$. Therefore, vascular SMCs may be a suitable target using adventitial deposition of gene therapy vectors $[3,9]$.

Viral and non-viral vector systems have been developed for gene transfer (GT). Viral vectors are powerful vehicles for GT into somatic cells [10]. Adenoviral vectors have been widely used in vascular GT. However, such gene carriers based on viral vectors are highly immunogenic, relatively difficult to handle compared with plasmids and require enhanced biosafety levels. In contrast, non-viral vectors are cost-efficient, easy to handle and hardly induce any immune response. Furthermore, no specific infrastructure is necessary. Non-viral vector systems are usually composed of either 'naked' DNA or DNA complexed with amphipathic complexing agents to achieve higher transfer efficiencies [11].

During the past years, liposome-mediated, non-viral GT has been considerably improved by the introduction of new cationic lipid formulations [12]. Cationic lipids condense DNA through ionic interactions, thereby forming lipoplexes [13]. Despite such improvements, non-viral GT, in particular transfections of stationary or low proliferating cells, remained rather inefficient [13]. Nevertheless, optimization of GT conditions using cationic lipids led to significantly improved transfection rates in cultures of proliferating or stationary primary vascular cells as well as in vivo in a porcine restenosis model [14]. Yet, GT efficiency still needs further improvement to achieve high levels of gene expression for clinical use. One of the limitations for sufficient levels of gene expression is the relatively inefficient release of DNA from endosomes into the cytoplasm and the limited protection of DNA against cytoplasmic nucleases allowing for high amounts of intact DNA to enter the nucleus. Promising DNA-condensing agents are cationic polymers like polylysines, polyarginines, spermine, spermidine, dendrimers and polyethylenimine [15]. These polymers are believed to still adhere to DNA after endosomal release, thus allowing for enhanced protection of the DNA against cytoplasmic nucleases. Poly-L-lysines were already used to condense DNA into a virus-like structure [16]. However, they alone are rather inefficient in promoting GT. Furthermore, their toxicity significantly increases with molecular weight [17].

Promising alternatives are lipopolyplexes, i.e. the combination of cationic lipids and cationic polymers, e.g., poly-L-lysine. Plasmid DNA complexed with poly-Llysine of $23.8 \mathrm{kDa}$ (163 lysine residues) and liposomes allowed for significantly improved GT [18]. However, short poly-L-lysines (sPLLs) of 13-18 lysine residues are characterized by already sufficient DNA binding capacity but low toxicity [19]. Therefore, we investigated the capability of two sPLLs - L18 with 18 L-lysine residues and low molecular weight poly-L-lysine (LMW-L) which is a mixture of poly-L-lysines containing 7-30 L-lysine residues - in enhancing liposome-mediated transfection. Furthermore, GT experiments were performed in primary porcine SMCs representing more suitable cell culture models better mimicking the in vivo conditions.

\section{Materials and Methods}

DNA Plasmid, Liposomes and Adjuvants

A $\beta$-galactosidase reporter gene vector $\mathrm{pCMV} \beta(7.2 \mathrm{~kb}$, cytomegalovirus-driven galactosidase gene; Clontech, Heidelberg, Germany) and the cationic lipid DOCSPER [1,3-dioleoyloxy-2( $\mathrm{N}^{5}$-carbamoyl-spermine)-propane] [20] were used for transfections. LMW-L (1-4 kDa) was purchased at Sigma-Aldrich (Munich, Germany) and L18 (2.6 kDa) was synthesized to a purity $>95 \%$ by Dr. Arnold (Gene Center, Munich, Germany). Both are referred to as sPLLs.

\section{Complex Formation}

Lipopolexes or polyplexes were prepared as follows: first, plasmid DNA (pCMV $\beta$ ) and DOCSPER or poly-L-lysine were diluted in separate tubes using either OptiMEM (Gibco, Eggenstein, Germany; high ionic strength solution) or deionised water (low ionic strength solution). Cationic lipid or polymer dilution was then added to the DNA tube at different ratios (DOCSPER/DNA 1/1$12 / 1$; sPLL/DNA $1 / 1-50 / 1$; w/w), mixed and incubated at room temperature for 30-40 min to allow for complex formation. In the case of lipopolyplexes, DNA was first pre-complexed with sPLLs by adding the polymer at different ratios directly into the tube with plasmid DNA, mixed and incubated for 5-15 min prior to the addition of the diluted DOCSPER again at various ratios and incubated at room temperature for additional 30-40 min.

\section{Particle Size Measurement}

Particle size was determined using dynamic light scattering with Zetasizer 3000HS (Malvern Instruments, Malvern, UK) using 10 subruns for each measurement in a $400-\mu l$ complex solution volume. Complexes were prepared as for GT and diluted either in low or high ionic strength solution (deionized water or OptiMEM). Formulations were analysed by measuring particle size in 10-min intervals for up to $60 \mathrm{~min}$ following the complex formation.

Resistance against DNase I Degradation

Lipoplexes and lipopolyplexes were prepared in $400 \mu \mathrm{lOp}$ tiMEM as described above using $2.5 \mu \mathrm{g} / \mathrm{ml}$ of plasmid DNA. Following complex formation, $400 \mu \mathrm{l}$ DNase I solution, i.e. $60 \mathrm{U} / \mathrm{ml}$ 
DNase I (Promega, Munich, Germany) and $1 \mathrm{mM} \mathrm{MgCl}_{2}$ in Hanks' balanced salt medium, was added and complexes were incubated at $37^{\circ} \mathrm{C}$ for $0,15,30,45$ and $60 \mathrm{~min}$. The enzyme reaction was terminated by addition of stop solution (Promega) and incubation at $65^{\circ} \mathrm{C}$ for $10 \mathrm{~min}$. Then, sodium dodecyl sulphate was added to a final concentration of $10 \%$ to release DNA from the condensing reagents, and $20 \mu \mathrm{l}$ of samples were loaded on a $0.8 \%$ agarose gel. The integrity of DNA in each formulation was compared with untreated DNA as a control.

\section{Cell Cultures}

Primary porcine SMCs were isolated from explants of porcine aorta $[4,21]$. The outgrowing SMCs were tested by immunohistochemistry for $\alpha$-smooth muscle actin expression [21]. The cells were cultured in medium (Ham's F-12/Waymouth 1/1; Gibco) containing $10 \%$ fetal calf serum, $4 \mathrm{~mm}$ glutamine, $100 \mathrm{U}$ penicillin, $100 \mu \mathrm{g} / \mathrm{ml}$ streptomycin and $2.5 \mathrm{mg} / \mathrm{ml}$ amphotericin B (Biochrom, Berlin, Germany) as previously described [4].

\section{Transfection in vitro}

The cells were seeded into 12 - or 96 -well plates $24 \mathrm{~h}$ prior to transfection. For the inhibition of SMC proliferation, cells were incubated with $300 \mu \mathrm{g} / \mathrm{ml}$ heparin (Braun, Melsungen, Germany) for further $24 \mathrm{~h}$ before transfection and following each medium change [14]. GT by using the cationic lipid DOCSPER was performed as described previously [14, 22]. Four hours following complex addition, transfection medium was replaced with fresh growth medium, and after an additional 2 days, GT efficiency was determined by measuring $\beta$-galactosidase activity.

\section{Transfection in vivo}

Optimal transfer conditions found in cell cultures were used for local gene delivery in a pig model using needle injection catheter as described previously [4, 12, 21, 23, 24]. Briefly, the arterial access was gained through carotid artery. X-ray contrast angiography was performed to guide the insertion and to make sure that the needle injection catheter (Bavaria Medicine Technology, Oberpfaffenhofen, Germany) is in the desired position at the site of the femoral artery. When in position, the fine needles were extended to penetrate the arterial wall into the perivascular tissue, and the transfection medium was deposited by a special injection pump place at the distal end of the catheter. This procedure ensures deposition of transfection solution within a region of about $2-3 \mathrm{~cm}$ diameter around the vessel into the adventitial tissue. After delivery of transfection medium, needles were retracted and the catheter was removed. All animal procedures were approved by the Animal Care and Use Review Committee of the University of Marseille according to French guidelines. The investigation conforms to the Guide for the Care and Use of Laboratory Animals published by the US National Institutes of Health (NIH publication No. 85-23, revised 1996). Study animals were Pietrain pigs (average weight $35 \mathrm{~kg}$ at the initial procedure; $\mathrm{n}=6$ ). In each femoral artery transfection was performed with $30 \mu \mathrm{g}$ plasmid DNA complexed with $300 \mu \mathrm{g}$ DOCSPER or precomplexed with poly-L-lysine for 5-15 min and then mixed with DOCSPER in $2 \mathrm{ml}$ OptiMEM (Gibco). Animals were sacrificed 3 weeks following transfection, and the $4-\mathrm{cm}$-long specimens of each femoral artery were surgically dissected in the way that the transfection site was located in the middle of the specimen. The explanted artery was then subdivided into 8 equal segments and marked from distal to proximal as S1 to S8. Samples 1, 3, 5 and 7 of each artery were snap-frozen in fluid nitrogen and used for DNA and expression analysis. Segments 2, 4, 6 and 8 were used for histological analyses (data not shown). Persons performing transfection in vivo, the extraction of specimens and the expression analysis were unaware of the deposition of the transfection vectors. $\beta$-galactosidase activity was measured as previously described [23].

\section{Fluorescent Staining, Flow Cytometry and Confocal \\ Microscopy}

For flow cytometric analysis, proliferating SMCs were seeded into 12 -well plates and cultivated as previously described $[9,14$, 22]. Transfection with Cy5-labelled (excitation at $650 \mathrm{~nm}$, emission at $670 \mathrm{~nm}$ ) plasmid DNA (Mirus Bio Corporation, Madison, Wisc., USA), unlabelled sPLLs and DOCSPER was done as described above. Four hours following gene delivery, the transfection medium was removed, cells were washed with phosphatebuffered saline (PBS) and treated with $500 \mu \mathrm{g} / \mathrm{ml}$ heparin for $30 \mathrm{~min}$ at $37^{\circ} \mathrm{C}$ to remove extracellular bound complexes. Subsequently, cells were trypsinised and measurement was performed with FACSCalibur (Becton Dickinson, Biosciences, San Jose, Calif., USA).

For colocalisation of sPLLs and plasmid DNA inside the cell, frequency resonance energy transfer (FRET), confocal microscopy and two assays from Molecular Probes (Madison, Wisc., USA) were used: Alexa Fluor $488(494 \mathrm{~nm} / 519 \mathrm{~nm})$ protein labelling kit for labelling of sPLLs and Label IT TM-Rhodamine $(546 \mathrm{~nm} / 576 \mathrm{~nm})$ labelling kit for DNA. Stationary SMCs were seeded on Lab-Tek 8-well chambered cover glasses (Nalge Nunc International, Naperville, Ill., USA) $24 \mathrm{~h}$ prior to transfection. Four and $24 \mathrm{~h}$ following transfection, cells were washed with PBS, fixed with $4 \%$ paraformaldehyde, and nuclei were stained with DAPI fluorochrome ( $1 \mu \mathrm{g} / \mathrm{ml}$ final concentration in PBS) for 5 min. DAPI staining solution was than replaced by PBS and samples were analysed using a confocal laser scanning microscope (LSM 510, Zeiss, Jena, Germany).

\section{Analysis of Toxicity}

For the determination of toxicity, the CellTiter 96 Aqueous One Solution Cell Proliferation Assay (Promega) was used. Two days following incubation with SPLL-enriched cell culture medium in 96-well plates, cells were washed with PBS, $100 \mu \mathrm{l}$ fresh growth medium containing $20 \mu \mathrm{l}$ of tetrazolium compound (MTS) was added (final concentration: $33 \mu \mathrm{g} / \mathrm{ml} \mathrm{MTS,} 25 \mu \mathrm{g} / \mathrm{ml}$ phenazine methosulphate), and the 96 -well plate was incubated at $37^{\circ} \mathrm{C} / 5 \% \mathrm{CO}_{2}$ for an additional $2 \mathrm{~h}$. The tetrazolium compound of the assay is bioreduced by cells into a coloured formazan product that is soluble in tissue culture medium and corresponds to the amount of metabolically active cells, and the optical absorbance is directly proportional to the number of living cells in the culture. The colour development was determined using an ELISA reader (Titertek Multiscan MCC 340, Eflab, Helsinki, Finland) at $490 \mathrm{~nm}$, with a reference at $690 \mathrm{~nm}$. The cellular toxicity of cells treated with the raising concentration of sPLLs was expressed in percent of non-treated cells. As a positive control (100\%), samples without sPLLs treatment were used $(0 \mu \mathrm{g} / \mathrm{ml} \mathrm{sPLL})$. The negative control $(0 \%)$ presented samples without cells containing growth medium and tetrazolium compound. 
Table 1. Particle size (nm) of sPLL/ DNA complexes $30 \mathrm{~min}$ following complexation

\begin{tabular}{llcrrr}
\hline \multirow{2}{*}{ Solution } & \multirow{2}{*}{ SPLL } & \multicolumn{3}{l}{ sPLL/DNA ratio } & \\
\cline { 3 - 6 } & & $1 / 1$ & $5 / 1$ & $10 / 1$ & \multicolumn{1}{c}{$50 / 1$} \\
\hline Water & L18 & $108 \pm 29$ & $62 \pm 18$ & $58 \pm 17$ & $43 \pm 13$ \\
& LMW-L & $295 \pm 13$ & $75 \pm 17$ & $64 \pm 21$ & $52 \pm 14$ \\
\hline OptiMEM & L18 & $2,206 \pm 976^{*}$ & $2,067 \pm 398^{*}$ & $1,672 \pm 242^{*}$ & $1,077 \pm 443^{*}$ \\
& & $263 \pm 71^{* *}$ & $242 \pm 65^{* *}$ & $233 \pm 33^{* *}$ & $219 \pm 87^{* *}$ \\
& LMW-L & $1,134 \pm 387^{*}$ & $1,456 \pm 421^{*}$ & $1,597 \pm 266^{*}$ & $1,689 \pm 348^{*}$ \\
& & $323 \pm 119^{* *}$ & $292 \pm 87^{* *}$ & $267 \pm 126^{* *}$ & $190 \pm 46^{* *}$ \\
\hline
\end{tabular}

Large- $\left({ }^{*}\right)$ and small-sized particles $\left(^{* *}\right)$ of these complexes were observed.

Table 2. Particle size ( $\mathrm{nm}$ ) of lipoplexes of DOCSPER and lipopolyplexes of DOCSPER/sPLLs

\begin{tabular}{lccccr}
\hline Weight ratio & $10 \mathrm{~min}$ & $20 \mathrm{~min}$ & $30 \mathrm{~min}$ & $40 \mathrm{~min}$ & $60 \mathrm{~min}$ \\
\hline DOCSPER/DNA (6/1) & $2,180 \pm 409^{*}$ & $\begin{array}{c}1,850 \pm 340 \\
211 \pm 39\end{array}$ & $\begin{array}{c}1,730 \pm 321 \\
265 \pm 14\end{array}$ & $\begin{array}{c}1,892 \pm 172 \\
238 \pm 41\end{array}$ & $\begin{array}{c}2,299 \pm 454 \\
221 \pm 41\end{array}$ \\
\hline DOCSPER/LMW-L/DNA (6/50/1) & $1,289 \pm 133$ & $1,518 \pm 106$ & $1,937 \pm 178$ & $1,606 \pm 134$ & $1,367 \pm 94$ \\
& $143 \pm 14$ & $221 \pm 18$ & $386 \pm 18$ & $264 \pm 38$ & $187 \pm 47$ \\
\hline DOCSPER/L18/DNA (6/10/1) & $1,897 \pm 456$ & $1,670 \pm 377$ & $1,562 \pm 324$ & $1,684 \pm 382$ & $1,051 \pm 272$ \\
& $178 \pm 33$ & $162 \pm 34$ & $158 \pm 24$ & $154 \pm 37$ & $109 \pm 14$ \\
\hline
\end{tabular}

Two subpopulations were observed: large $\left(^{*}\right)$ and small sized $\left({ }^{* *}\right)$.

Statistical Analysis

Results are means \pm standard deviation (SD) of a minimum of 3 experiments for each condition. One-way ANOVA was used for comparison among groups (SPSS Inc., Chicago, Ill., USA). Statistical significance was defined as $\mathrm{p}<0.05$.

\section{Results}

\section{Complex Formation and Particle Size Measurement}

First, the sizes of sPLL/DNA particles forming at low and high ionic strength were measured (table 1). In solutions of low ionic strength and at sPLL/DNA ratios $>1 / 1$, both L18 and LMW-L formed small complexes $<100 \mathrm{~nm}$ in size. These formulations tended to form more tight complexes with decreasing size when the sPLL/DNA weight/weight $(\mathrm{w} / \mathrm{w})$ ratio was further increased. In general, the particle sizes tended to decrease with increasing sPLL/DNA ratios in low ionic strength solution. At high ionic strength (OptiMEM), both L18 and LMW-L, independent of the weight ratio used, formed particles with
DNA $>1 \mu \mathrm{m}$ : up to $2,206 \pm 976 \mathrm{~nm}$ for L18/DNA and up to $1,789 \pm 348 \mathrm{~nm}$ for LMW-L/DNA. However, small complexes ranging from $190 \pm 46$ to $323 \pm 119 \mathrm{~nm}$ were also observed (table 1). To determine the time-dependent formation and sizes of different lipopolyplex formulations, a L18/DNA ratio of 5/1 and a LMW-L/DNA ratio of 50/1 were selected, and the particle sizes were compared with those of lipoplexes of DOCSPER (table 2). Again, two subpopulations, one of large particles with sizes of up to 2,180 $\pm 409 \mu \mathrm{m}$ and one of small particles between $109 \pm 14$ and $386 \pm 18 \mathrm{~nm}$, were identified. There were no significant differences in size between the lipoplexes and lipopolyplexes. All types of aggregates already formed during the first $10 \mathrm{~min}$ following mixing of the components. For up to $1 \mathrm{~h}$, none of the complexes displayed significant changes in particle size (table 2).

\section{Resistance against DNase I Degradation}

To analyse the ability of sPLLs to protect DNA from degradation, complexes of sPLLs with plasmid DNA were 
Fig. 1. Resistance of DNA against DNase I degradation in complexes with sPLLs (L18 or LMW-L) (a) or sPLLs and DOCSPER (lipopolyplexes) in comparison with DNA in lipoplexes of DOCSPER (b). Polyplexes of sPLL/DNA or lipopolyplexes of DOCSPER/sPLL/DNA were formed as described in Materials and Methods. DOC = DOCSPER/DNA formulation.

Table 3. Internalisation of lipoplexes of DNA with DOCSPER and lipopolyplexes of DNA with L18 or LMW-L into SMCs at different time points using Cy5 fluorescently labelled plasmid DNA and flow cytometry

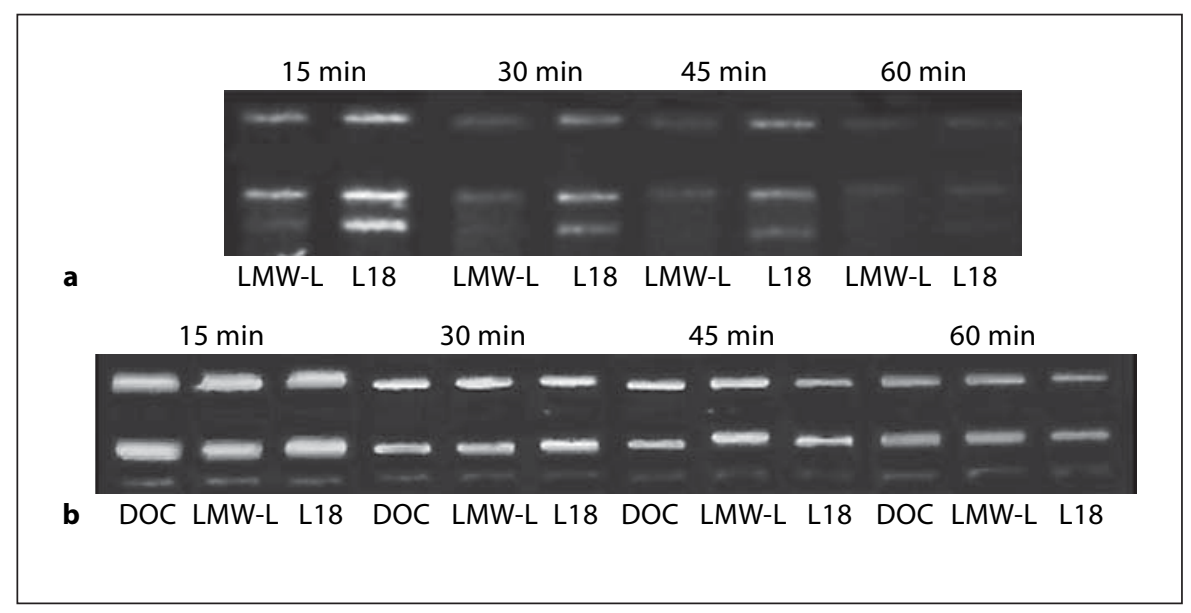

\begin{tabular}{lrrr}
\hline & \multicolumn{1}{c}{$2 \mathrm{~h}$} & \multicolumn{1}{c}{$4 \mathrm{~h}$} & \multicolumn{1}{c}{$24 \mathrm{~h}$} \\
\hline Naked DNA & $2.65 \pm 0.86$ & $4.25 \pm 1.78$ & $0.25 \pm 0.42$ \\
DOCSPER/DNA & $58.60 \pm 1.13$ & $79.90 \pm 5.23$ & $98.15 \pm 2.05$ \\
DOCSPER/L18/DNA & $30.65 \pm 1.06$ & $61.75 \pm 6.86$ & $98.50 \pm 0.99$ \\
DOCSPER/LMW-L/DNA & $30.25 \pm 3.04$ & $63.85 \pm 9.40$ & $98.50 \pm 0.85$ \\
\hline
\end{tabular}

The same conditions for complex formation were used as described in table 2. Uptake efficiency is indicated as percentage of all cells. For detecting internalised complexes, cells were treated with heparin prior to measurement (see also Materials and Methods). treated with DNase I (fig. 1). Both types of sPLLs protected plasmid DNA against nuclease degradation for up to 45 min (fig. 1a). In complexes with L18, the DNA displayed a slightly higher resistance against DNase I than in those with LMW-L. However, after $60 \mathrm{~min}$ of incubation of sPLL/DNA polyplexes with DNase I, plasmid DNA was almost completely degraded (fig. 1a). In contrast, lipopolyplexes of sPLLs and DOCSPER were stable against degradation for at least $60 \mathrm{~min}$ following DNase I addition (fig. 1b). Uncomplexed DNA was digested within the first minutes following DNase I addition (data not shown).

\section{Cellular Uptake and Intracellular Colocalization}

Plasmid DNA was fluorescently labelled with Cy5 and used to examine the internalisation of transfection complexes into SMCs. Flow cytometric analysis of cellular uptake following addition of lipoplexes with DOCSPER and lipopolyplexes containing DOCSPER and L18 or LMW-L was carried out after 2, 4 and $24 \mathrm{~h}$ (table 3 ). Results demonstrated no difference in cellular uptake independent of the formulation used; only the velocity of the internalisation varied. Twenty-four hours following complex addition, all cells $(>98 \%)$ were positive for the transfected DNA. Lipoplexes of DOCSPER and DNA seemed to be internalised faster than lipopolyplexes, with more than $50 \%$ positive cells already $2 \mathrm{~h}$ following transfection. For lipopolyplexes, no significant difference in the velocity of internalisation was observed between complexes with L18 or LMW-L. In contrast, the uptake of naked DNA was very low with up to $4 \%$ after $4 \mathrm{~h}$. At $24 \mathrm{~h}$, no significant amount of naked DNA was detected inside the cells (table 3).

Intracellular colocalisation of DNA and sPLLs after 4 and $24 \mathrm{~h}$ was analysed by the detection of FRET using confocal microscopy (fig. 2). Following the addition of double-labelled L18/DNA or LMW-L/DNA formulations to SMCs, the excitation of tetramethyl (TM)-rhodaminelabelled DNA caused the emission of AlexaFluor (sPLL labelling) in all cases indicating the colocalisation of DNA and sPLLs up to $24 \mathrm{~h}$ following complex delivery. No differences in colocalisation results were observed between L18/DNA or LMW-L/DNA formulations used. 
Without FRET
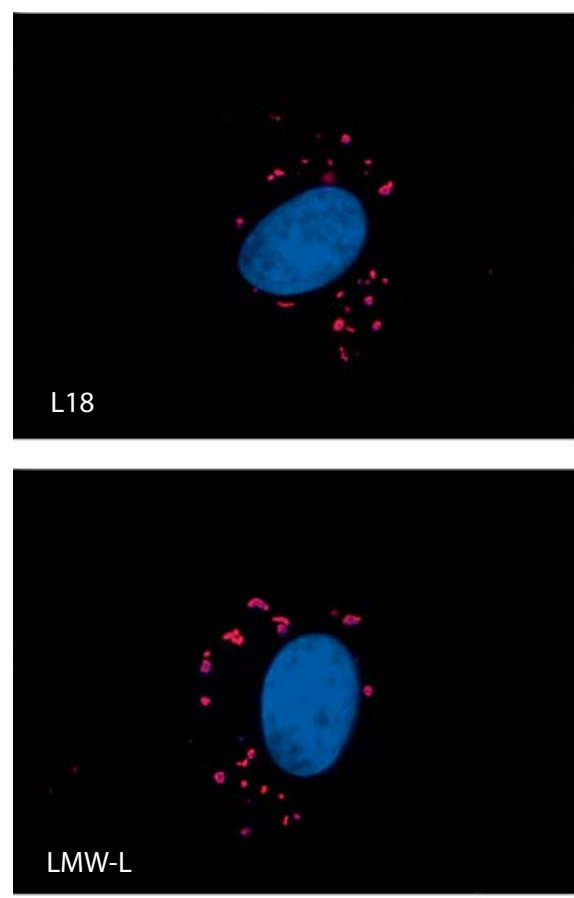

and sPLLs by FRET using confocal microscopy in stationary SMCs $4 \mathrm{~h}$ (a) and $24 \mathrm{~h}$ (b) following GT with lipopolypexes of DOCSPER/sPLL/DNA. DNA was labelled with TM-rhodamine and sPLLs with AlexaFluor 488 to enable FRET visualisation. Cell nuclei were stained with DAPI. Left panels: TM-rhodamine fluorescence emission after specific excitation of TM-rhodamine (emission at $546 \mathrm{~nm}$, excitation at $576 \mathrm{~nm}$; without FRET). Right panels: TM-rhodamine emission after excitation of AlexaFluor 488 (emission at 494 $\mathrm{nm}$, excitation at $519 \mathrm{~nm}$; with FRET).
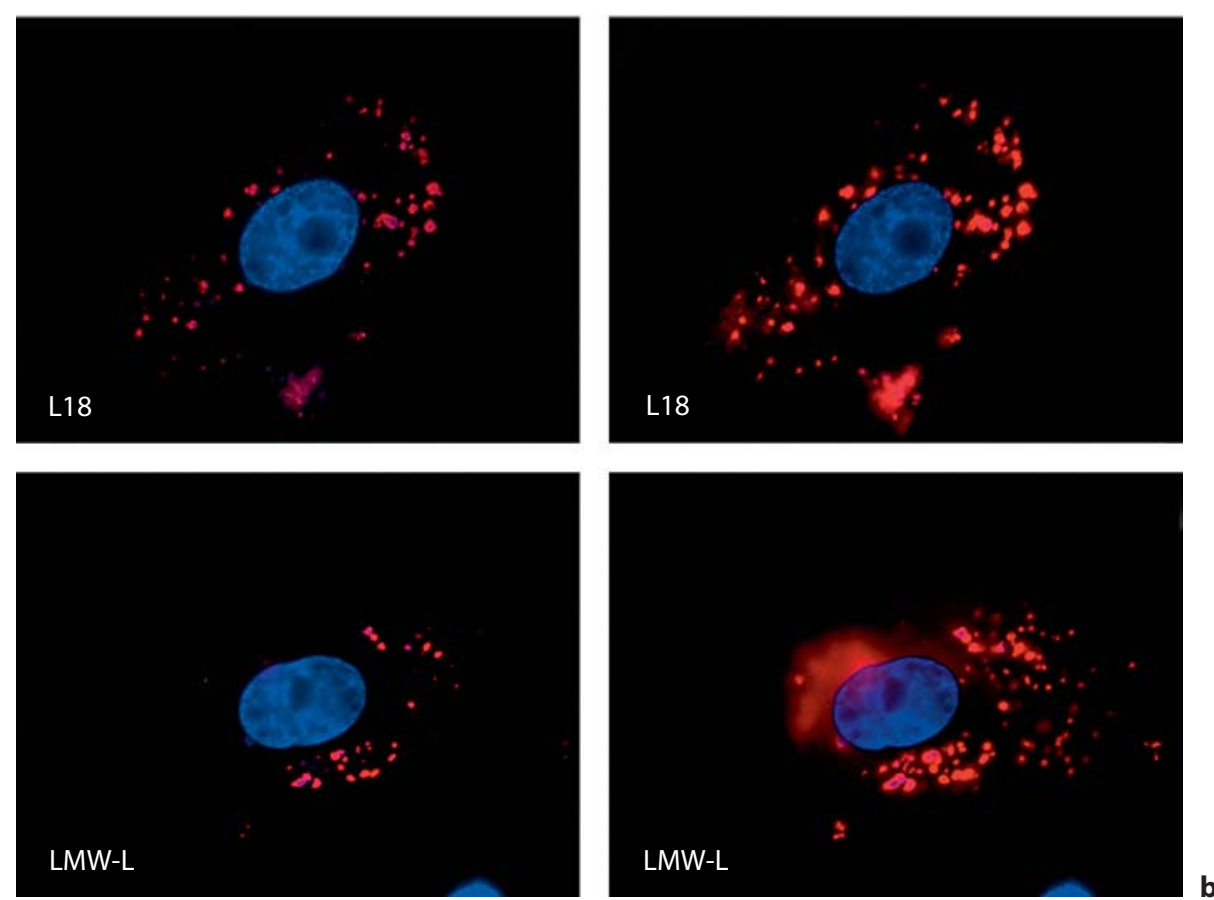

b
With FRET
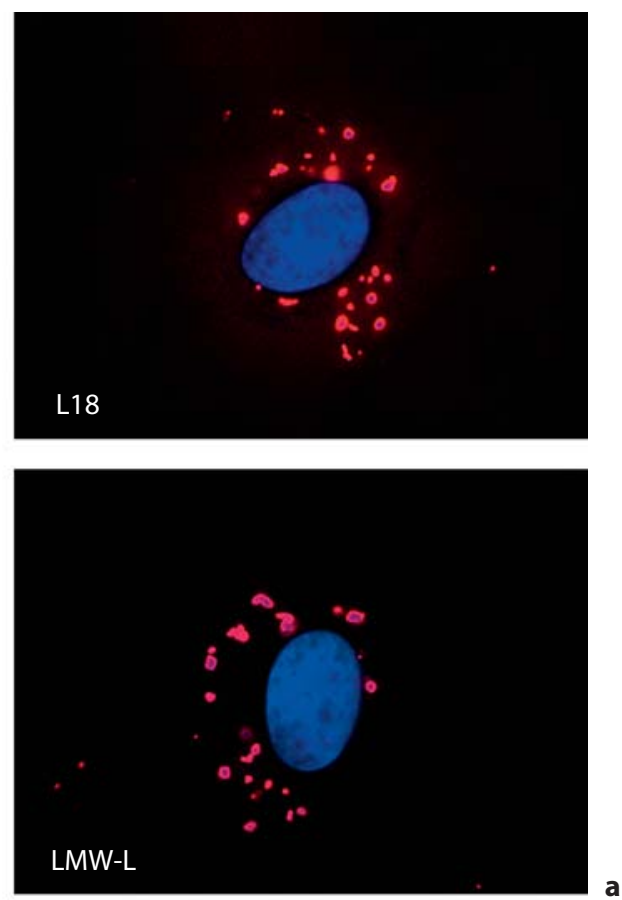

\section{Toxicity of sPLLs on SMCs}

To investigate the toxicity of sPLLs on SMCs, cells were incubated with increasing concentrations of L18 and LMW-L for up to $500 \mu \mathrm{g} / \mathrm{ml}$. After $24 \mathrm{~h}$ of incubation and up to the concentration of $100 \mu \mathrm{g} / \mathrm{ml}$ sPLLs, no toxic effects on SMCs were observed for both LMW-L and SPLL (fig. 3). This concentration was significantly higher than the concentration used in the transfection experiments. The synthetic poly-L-lysine L18 seemed to be less toxic than LMW-L; however, the differences in cell viability were not statistically significant. 


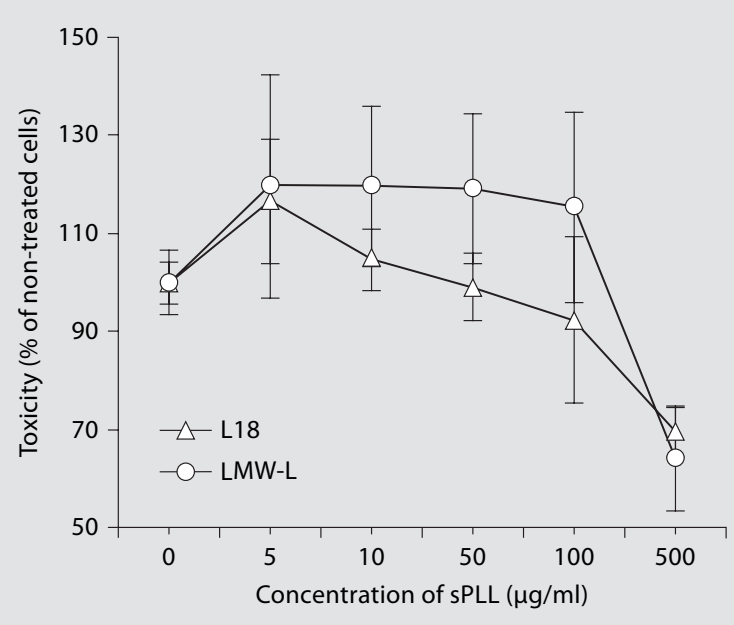

Fig. 3. Cytotoxicity of sPLLs on SMCs 1 day following treatment. The SMCs were incubated in growth medium containing diverse concentrations of sPLL to examine the toxic effect of the sPLLs. Non-treated cells were set as $100 \%$, whereas samples without cells containing only growth medium and tetrazolium compound were set as $0 \%$. The values are means \pm SD of triplicates.

\section{Transfection in vitro}

To analyse the effect of sPLLs as components of lipopolyplexes on GT efficiency, various amounts of sPLLs were used for transfection of proliferating and quiescent SMCs. As a control, GT with lipoplexes of DOCSPER without sPLLs was performed. The DOCSPER/DNA ratio used for proliferating cells was $6 / 1$ and for stationary SMCs 10/1 [14, 22]. In proliferating SMCs, lipopolyplexes containing L18 displayed an up to 2 -fold higher transfection efficiency than the corresponding lipoplexes. The maximal GT efficiency was achieved using $5-10 \mu \mathrm{g}$ of L18 and $1 \mu \mathrm{g}$ plasmid DNA per 100,000 cells (fig. 4a). Pre-incubation of DNA with LMW-L increased GT efficiency for up to 2.5-fold in comparison with the transfer efficiency of lipoplexes alone. Maximal GT was achieved using 50-100 $\mu \mathrm{g}$ LMW-L and $1 \mu \mathrm{g}$ plasmid DNA per 100,000 cells. Thus, to achieve maximal GT efficiency of lipopolyplexes with LMW-L, a 10-fold higher sPLL concentration was necessary compared with lipopolyplexes with L18. In stationary SMCs, L18 increased the GT efficiency up to 6 -fold at L18/DNA weight ratios of 5/1 and 10/1. At higher ratios up to $100 / 1$ (L18/DNA), the transfer efficiency remained almost constant. Lipopolyplexes with LMW-L displayed maximal GT at an LMW-L/DNA ratio of 50/1 (fig. 4b). In stationary SMCs, L18 and LMW-L lipopolyplexes exhibited comparable maximal GT efficiencies.
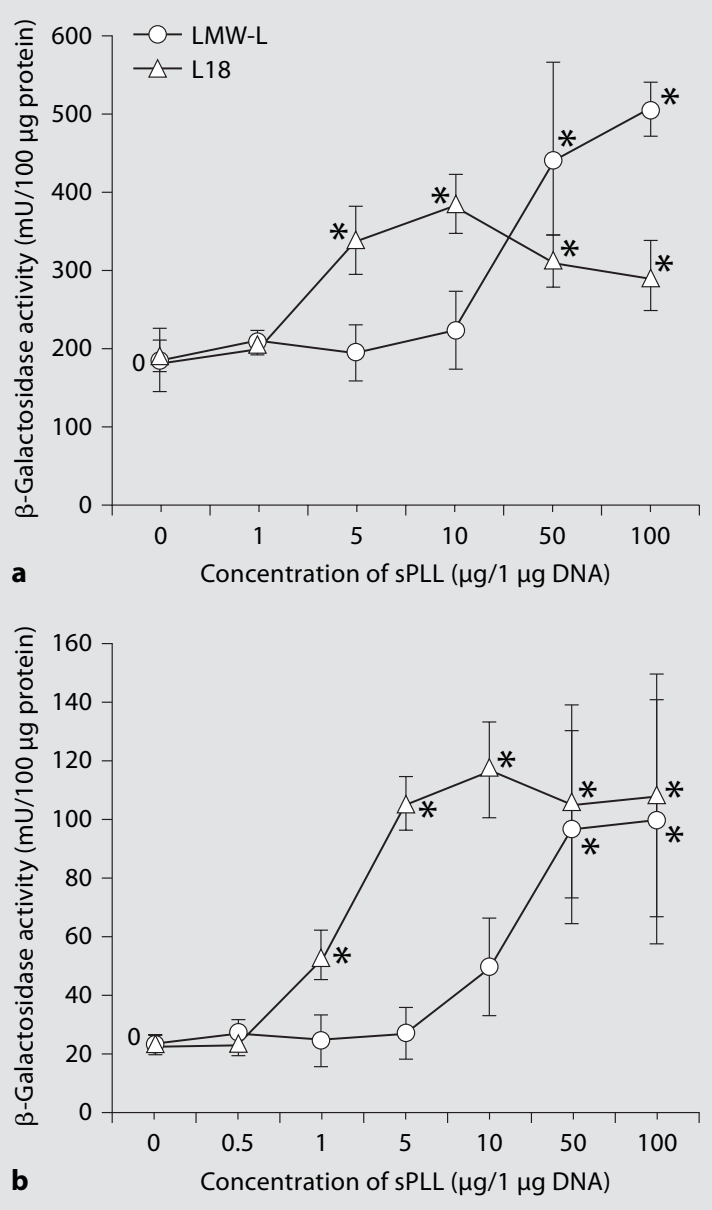

Fig. 4. Dependency of transfection efficiency expressed as $\beta$-galactosidase activity on the concentration of sPLLs in lipopolyplexes used for gene transfer of a $\beta$-galactosidase-encoding DNA plasmid in proliferating (a) or quiescent (b) porcine SMCs. * Significant enhancement of $\beta$-galactosidase activity of lipopolyplexes comprising sPLL in comparison with lipoplexes of DOCSPER $(0=$ without $\mathrm{sPLL})$.

\section{Transfection in vivo}

The effects of lipopolyplexes on transfection were also analysed in vivo in a porcine model. Since a significant lower concentration of L18 than of LMW-L was required in vitro for maximal GT efficiency $(5-10 \mu \mathrm{g} \mathrm{L18} \mathrm{vs} .50 \mu \mathrm{g}$ LMW-L per $1 \mu \mathrm{g}$ plasmid DNA and 100,000 cells), L18 was chosen as sPLL for in vivo experiments. The ratio of DOCSPER/DNA lipoplexes used was 10/1 (w/w; optimal ratio found in GT experiments with stationary primary porcine SMCs in vitro) [14, 22]. For lipopolyplexes, the ratio of DOCSPER/L18/DNA was 10/10/1 (w/w/w). Three weeks following local application of L18 lipopolyplexes, 


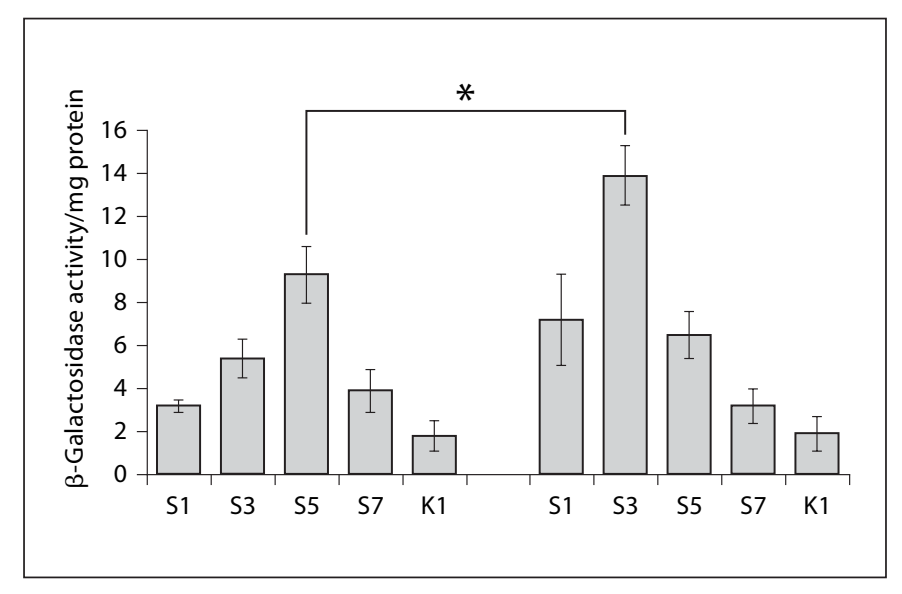

Fig. 5. $\beta$-galactosidase activity in porcine iliac arteries in vivo 3 weeks following gene transfer using DOCSPER/DNA lipoplexes (left) versus DOCSPER/L18/DNA lipopolyplexes (right). S1-S7 = Individual segments of the explanted femoral artery analysed separately for $\beta$-galactosidase activity; $\mathrm{K} 1$ = non-transfected control tissue. ${ }^{*} \mathrm{p}<0.05$.

up to 1.5 -fold higher $\beta$-galactosidase activities were observed in protein extracts from arterial segments as compared with the application of lipoplexes of DNA and DOCSPER (fig. 5).

\section{Discussion}

This study was designed to reveal a possible advantage of the use of sPLL L18 (2.6 kDa, 18 lysine residues) or LMW-L (1-4 kDa, 7-30 lysine residues) in liposomal gene delivery in cultures of primary porcine SMCs in vitro and in vivo in a porcine femoral artery using local delivery. In addition to the analysis of GT efficiencies, the influences of the sPLLs L18 and LMW-L on liposome/DNA complex formation, stability, resistance against degradation by nucleases, uptake into the cells and visualisation of the complexes inside the cells were tested.

Biophysical properties such as the size of DNA complexes play an important role in GT efficiency $[25,26]$. Therefore, the sPLL/DNA complexes were first characterised with regard to particle size. Both L18 and LMW-L formed small complexes with DNA at low ionic strength. However, at the sPLL/DNA ratio of $1 / 1$, complexes with L18 were significantly smaller than those with LMW-L. At ratios of 5/1 and higher, the sizes of both types of complexes were similar. These results can be explained by the fact that LMW-L contains a mix of lysine chains of dif- ferent lengths (7-30 lysine residues). Poly-L-lysines with 13 or less lysine repeats bind DNA weakly and produce large DNA condensates [19]. Thus, LMW-L contains lower amounts of poly-L-lysines with high DNA binding capacity at the same concentration as L18. Therefore, higher amounts of LMW-L are necessary to reach the same degree of DNA condensation. At high ionic strength, aggregates were observed for both sPLLs independent of the sPLL/DNA ratio. The appearance of two subpopulations coincided with the measured high polydispersity of complexes.

Similar to the corresponding lipoplexes, lipopolyplexes of sPLLs, DNA and DOCSPER displayed particle sizes $>1 \mu \mathrm{m}$ already $10 \mathrm{~min}$ following mixing of components at high ionic strength. No significant differences in particle size or complex-forming kinetics were observed between the lipopolyplexes with L18 or LMW-L and lipoplexes of DOCSPER. Consequently, the high ionic strength solution used for transfection should not significantly contribute to differences in transfer efficiency observed for the various formulations.

The uptake of GT particles into cells is another important factor influencing transfection efficiency [27] and was therefore comparatively investigated for lipopolyplexes containing sPLLs and DOCSPER and the lipoplex with DOCSPER. The experiments revealed that DOCSPER/DNA lipoplexes were internalised faster than lipopolyplexes. However, the amount of complexes finally internalised after $24 \mathrm{~h}$ did not differ between lipoplexes and lipopolyplexes, suggesting that the enhanced GT efficiency found for lipopolyplexes was not due to an enhanced uptake of these formulations into the cells. These data confirm the presumption that the cellular uptake of the DNA complexes alone does not give any evidence about the efficiency of GT. In addition to the successful internalisation, endosomal release, protection against degradation inside the cell and effective translocation in the nucleus are additional necessary properties of an ideal DNA carrier to succeed in GT [22, 25-27]. In contrast to the internalisation of lipoplexes, polyplexes or lipopolyplexes, the uptake of naked DNA was very low, and $24 \mathrm{~h}$ following treatment, no significant DNA inside the cells was detectable. This again confirms the fast degradation of unprotected DNA outside and also inside the cells, thus showing that naked DNA is not suitable for transfection.

Following endosomal release of DNA into the cytoplasm, its degradation by nucleases may significantly diminish transfection efficiency. In previous experiments, it was demonstrated that complexation of plasmid DNA 
with poly-L-lysine of a molecular weight of $25 \mathrm{kDa}$ efficiently protects DNA from DNase I digestion [28]. Our results demonstrated that polyplexes of L18/DNA displayed a slightly higher resistance against DNase I than LMW-L/DNA aggregates. This result can be again explained by a better complexation of DNA by L18 compared with LMW-L due to the defined length of L18 in contrast to LMW-L. However, the slightly lower capability of LMW-L to protect DNA from DNase I degradation did not affect the stability of DNA in the corresponding lipopolyplexes in comparison with lipopolyplexes containing L18 and lipoplexes containing only DOCSPER, suggesting that the latter is the component predominantly affecting the stability of DNA in lipopolyplexes.

Using the FRET technique which enables detection of colocalisation of two molecules inside the cell demonstrated that up to $24 \mathrm{~h}$ following GT, sPLLs were still bound to DNA, suggesting that DNA is protected from degradation by cytoplasmic nucleases after endosomal release. Consequently, this would enhance the probability for DNA to reach the nucleus and to become expressed and may therefore be the key mechanism responsible for the higher transfection rates of lipopolyplexes in comparison with lipoplexes.

Additionally or alternatively, the higher GT efficiency achieved by lipopolyplexes with sPLLs versus lipoplexes may be due to a higher degree of toxicity. Increased toxicity often contributes to enhanced transfection efficacy [22]. However, investigation of cytotoxicity of the formulations did not reveal any significant decrease in cell viability for up to $100 \mu \mathrm{g} / \mathrm{ml}$, which was far beyond our working concentration of sPLLs. In previous studies, poly-L-lysine with a molecular weight of $25 \mathrm{kDa}$ was found to improve GT in a number of cell lines in vitro [18, 29]. However, high molecular weight poly-L-lysine is significantly more toxic than the low molecular weight forms which are almost non-toxic. The concentrations of poly-L-lysine used in our experiments had no effect on cell proliferation or metabolic activity.

Using lipopolyplexes of sPLLs and DOCSPER under optimised GT conditions, sPLLs enhanced transfection rates not only in proliferating but also in stationary primary SMCs and improved GT up to 5-fold. The enhancement of GT efficiency in stationary or low proliferating cells is particularly important since the proliferation rates in our restenosis in vivo model systems are rather low and correspond well with the proliferation rates found in stationary cell cultures established in our previous studies by using heparin for growth inhibition [14]. No significant differences in GT efficiency were observed indepen- dently of the sPLLs used for lipopolyplex transfection. However, for lipopolyplexes with L18 in comparison with LMW-L, an about 10-fold lower concentration was already sufficient to achieve maximal GT effect. These discrepancies can be explained by the fact that LMW-L is a mixture of poly-L-lysines varying in chain length, which display different DNA binding affinities.

$\mathrm{Li}$ and Huang [30] showed that cationic lipid-protamine-DNA complexes were highly efficient in in vivo GT in a mouse model. However, so far, there was no evidence for an effect of poly-L-lysine pre-condensation on liposomal transfection in vivo. In the present paper, a supporting effect of sPLLs on transfection in vivo is demonstrated for the first time using a porcine experimental model. L18 was chosen for these experiments since its defined length presumably enables the application of a significantly lower concentration for maximal effect as it can be supposed for LMW-L. An up to 1.5-fold higher GT efficiency was observed for DOCSPER/L18/DNA lipopolyplexes compared with DOCSPER/DNA lipoplexes.

In summary, both low molecular weight poly-L-lysines, LMW-L and L18, significantly increased transfection efficiency measured by the activity of a reporter gene product. Biophysical properties and cellular uptake of DOCSPER lipoplexes did not significantly differ from the properties and internalisation of lipopolyplexes containing DOCSPER and sPLLs. Therefore, we conclude that the enhancement of GT by sPLLs is mainly due to the enhanced protection of transgene DNA by sPLLs against nuclease degradation in the cytoplasm and to a potential synergistic effect of sPLL and cationic lipids on endosomal release. The improvement in transfection efficiency using sPLLs was also confirmed in vivo in a porcine restenosis model. Thus, lipopolyplexes containing sPLLs with up to 30 lysine residues, and particularly those containing L18, may be promising agents for improved liposomemediated GT into vascular cells.

\section{Acknowledgements}

We thank the German Heart Foundation (Deutsche Herzstiftung, F/25/03) for the possibility to perform our scientific work and Mr. Gropp from Bernina (Martinsried, Germany) for providing the cationic lipid DOCSPER for our experiments. Furthermore, we kindly appreciate the scholarships from GFPS e.V., College of Graduates of German Research Foundation (Deutsche Forschungsgemeinschaft, GRK 438) and German Academic Exchange Service (DAAD) for supporting A.G. in this project. 


\section{References}

1 Nikol S, Huehns TY: Preclinical and clinical experience in vascular gene therapy: advantages over conservative/standard therapy. J Invasive Cardiol 2001;13:333-338.

2 Nikol S, Pelisek J, Engelmann MG, Rolland $\mathrm{PH}$, Armeanu S: Prevention of restenosis using the gene for cecropin complexed with DOCSPER liposomes under optimized conditions. Int J Angiol 2000;9:87-94.

3 Nikol S, Huehns TY, Höfling B: Molecular biology and post-angioplasty restenosis. Atherosclerosis 1996;123:17-31.

4 Nikol S, Huehns TY, Krausz E, Armeanu S, Engelmann MG, Winder D, Salmons B, Hoefling B: Needle injection catheter delivery of the gene for an antibacterial agent inhibits neointimal formation. Gene Ther 1999;6:737-748.

5 Bauters C, Isner JM: The biology of restenosis. Prog Cardiovasc Dis 1997;40:107-116.

6 Höfling B, Huehns TY: Intravascular local drug delivery after angioplasty. Eur Heart J 1995;16:437-440.

7 Huehns TY, Gonschior P, Höfling B: Adventitia as a target for intravascular local drug delivery. Heart 1996;75:437-438.

8 Shi Y, Piniek M, Fard A: Adventitial remodeling after coronary aterial injury. Circulation 1996;93:340-348.

9 Pelisek J, Kuehnl A, Rolland PH, Mekkaoui C, Fuchs A, Walker GF, Ogris M, Wagner E, Nikol S: Functional analysis of genomic DNA, cDNA, and nucleotide sequence of the mature C-type natriuretic peptide gene in vascular cells. Arterioscler Thromb Vasc Biol 2004;24:1646-1651.

10 Guenzburg WH, Salmons B: Virus vector design in gene therapy. Mol Med Today 1997; 1:494-503.

11 Miller AD: Cationic liposome systems in gene therapy. Curr Res Mol Ther 1998;1: 494-503.
12 Armeanu S, Pelisek J, Krausz E, Fuchs A, Groth D, Curth R, Keil O, Quilici J, Rolland PH, Reszka R, Nikol S: Optimization of nonviral gene transfer of vascular smooth muscle cells in vitro and in vivo. Mol Ther 2000; 1:1-10.

13 Eastman SJ, Siegel C, Tousignant J, Scheule RK: Biophysical characterization of cationic lipid: DNA complexes. Biochim Biophys Acta 1997; 1325:41-62.

14 Pelisek J, Armeanu S, Nikol S: Quiescence, cell viability, apoptosis and necrosis of smooth muscle cells using different growth inhibitors. Cell Prolif 2001;34:305-320.

15 Mahato RI, Monera OD, Smith LC, Rolland A: Peptide-based gene delivery. Curr Opin Mol Ther 1999;1:226-243.

16 Sorgi FL, Bhattacharya S, Huang L: Protamine sulfate enhances lipid-mediated gene transfer. Gene Ther 1997;4:961-968.

17 Wolfert MA, Schacht EH, Toncheva V, Ulbrich K, Nazarova O, Seymour LW: Characterization of vectors for gene therapy formed by self-assembly of DNA with synthetic block co-polymers. Hum Gene Ther 1996;17: 2123-2133.

18 Vitiello L, Chonn A, Wasserman JD, Duff C, Worton RG: Condensation of plasmid DNA with polylysine improves liposome mediated gene transfer into established and primary muscle cells. Gene Ther 1996;3:396-404.

19 Wadhwa MS, Collard WT, Adami RC, Mc Kenzie DL, Rice KG: Peptide-mediated gene delivery: influence of peptide structure on gene expression. Bioconjug Chem 1991;8:8188 .

20 Groth D, Keil O, Schneider M, Reszka R: Transfection assay for dual determination of toxicity and gene expression. Anal Biochem 1998;258:141-143.

21 Huehns TY, Krausz E, Mrochen S, Schmid M, Engelmann MG, Esin S, Schrittenloher PK, Hofling B, Gunzburg WH, Nikol S: Neointimal growth can be influenced by local adventitial gene manipulation via a needle injection catheter. Atherosclerosis 1999;144: 135-150.
22 Pelisek J, Engelmann MG, Golda A, Fuchs A, Armeanu S, Shimizu M, Mekkaoui C, Rolland PH, Nikol S: Optimization of nonviral transfection: variables influencing liposome-mediated gene transfer in proliferating vs quiescent cells in culture and in vivo using a porcine restenosis model. J Mol Med 2002;11:724-736.

23 Pelisek J, Armeanu S, Nikol S: Evaluation of $\beta$-galactosidase activity in tissue in the presence of blood. J Vasc Res 2000;37:585-593.

24 Nikol S, Pelisek J, Engelmann MG, Rolland $\mathrm{PH}$, Armeanu S: Prevention of restenosis using the gene for cecropin complexed with DOCSPER liposomes under optimized conditions. Int J Angiol 2000;9:87-94.

25 Ogris M, Steinlein P, Kursa M, Mechtler K, Kircheis R, Wagner E: The size of DNA/ transferrin-PEI complexes is an important factor for gene expression in cultured cells. Gene Ther 1998;5:1425-1433.

26 Boussif O, Zanta MA, Behr JP: Optimized galenics improve in vitro gene transfer with cationic molecules up to 1,000 -fold. Gene Ther 1996;3:1074-1080.

27 Khalil IA, Kogure K, Akita H, Harashima H: Uptake pathways and subsequent intracellular trafficking in nonviral gene delivery. Pharmacol Rev 2006;58:32-45.

28 Capan Y, Woo BH, Gebrekidan S, Ahmed S, DeLuca PP: Stability of poly(L-lysine)-complexed plasmid DNA during mechanical stress and DNase I treatment. Pharm Dev Technol 1999;4:491-498.

29 Gao X, Huang L: Potentiation of cationic liposome-mediated gene delivery by polycations. Biochemistry 1996;35:1027-1036.

30 Li S, Huang L: In vivo gene transfer via intravenous administration of cationic lipidprotamine-DNA (LPD) complexes. Gene Ther 1997;4:891-900. 